\title{
Guidance of a Steerable Cannula Robot in Soft Tissue Using Preoperative Imaging and Conoscopic Surface Contour Sensing
}

\author{
Ray A. Lathrop, Member, IEEE, D. Caleb Rucker, Member, IEEE, and Robert J. Webster III, Member, IEEE
}

\begin{abstract}
Intraoperative surface contour sensing can enable the registration of high-resolution three-dimensional preoperative images for precise guidance of surgical robots. This is particularly useful for guiding steerable needles in soft tissues. In this paper we combine a new minimally invasive surface scanning technique based on conoscopic holography with a steerable active cannula robot. We experimentally demonstrate cannula tip placement to multiple physical points inside phantom tissue, which correspond to points specified in preoperative images - the input an eventual clinical system would obtain from the physician. While the image-guided steerable system we propose is broadly applicable to many kinds of surgery, one particular application of interest is in ablating large liver tumors, where it is beneficial for the ablator to be repositioned to multiple locations without being withdrawn from the organ.
\end{abstract}

\section{INTRODUCTION}

Robotic systems can demonstrably improve accuracy in interventional medical procedures requiring sampling from, or therapy delivery to, a specific site identified on preoperative images [1], [2]. In these systems, the robot accomplishes the translation of image space coordinates to patient space coordinates and then precisely aligns the needle with the desired location [3], [4] - a task robots can do more accurately and reliably than is possible with human memory, spatial reasoning, and hand-eye coordination [5].

To compensate for sources of error during insertion (e.g. small registration errors, tissue deformation), minimize the number of repeated needle insertions required, and enable access to targets without feasible straight-line trajectories, several strategies for needle steering have recently been developed. These include applying forces and torques to the base of a stiff symmetric-tip needle [6], [7], incorporating a precurved stylet within a straight cannula [8], harnessing bevel tip forces with flexible shafts [9], and employing multiple precurved tubes which change needle shaft shape as they are telescopically extended and axially rotated with respect to one another [10], [11].

Such systems are typically designed to operate under intraoperative Ultrasound (US) guidance [5], inside Computed Tomography (CT) scanners [12], under fluoroscopic guidance [13], and in conjunction with Magnetic Resonance Imaging (MRI) [14]. Each of these imaging modalities has its own strengths and weaknesses. Ultrasound is inexpensive and easily accessible, but provides low-resolution images.

R. A. Lathrop, D. C. Rucker, and R.J. Webster III are with Vanderbilt University, Nashville, TN 37235, USA (e-mail: ray.a.lathrop, daniel.c.rucker, robert.webster@vanderbilt.edu).

This material is based upon work supported in part by National Science Foundation grant 0651803 and in part by National Institute of Health grant R44 CA134169. Manuscript received September 15, 2009.

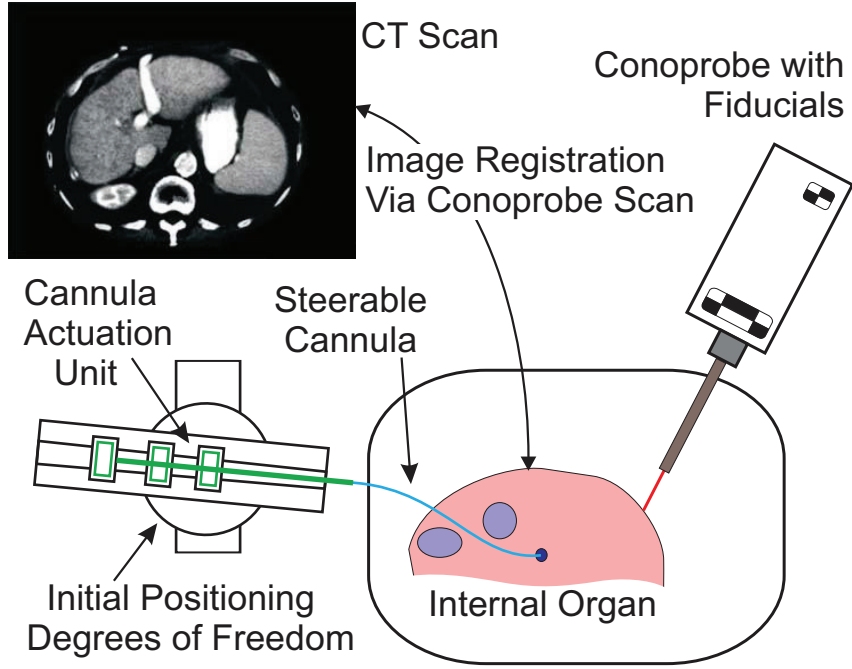

Fig. 1. Diagram of our Conoprobe/preoperative image-guided steerable cannula system. A segmented preoperative CT or MRI scan of an organ is registered to a surface scan collected in vivo via a tracked Conoprobe.

CT provides better images, but intraoperative use involves ionizing radiation, as does fluoroscopy. MRI provides excellent soft tissue discrimination, but is expensive, less widely available, and requires specialized robot hardware that is compatible with strong magnetic fields.

In order to make intraoperative use of the highest resolution images available including MRI without introducing additional ionizing radiation, surface-based techniques for registering images to physical coordinates have been developed [15]. These are necessary in soft tissues which generally lack identifiable surface features and cannot support the screw-based fiducial markers typically employed in bones. A contactless method of surface scanning is a laser range scanner (LRS) which triangulates a large number of points using a laser-based grid pattern [15]. LRS systems require line of sight, and hence wide exposure of the surgical site. Once a scan is collected, a variety of rigid and deformable registration techniques (e.g. [16], [17]) can be used to register preoperative images to physical coordinates.

Recently, conoscopic holography has been proposed as a means of accomplishing organ surface point cloud sensing in a minimally invasive setting [18]. In this system the Conoprobe, a 1D noncontact distance measuring device, is optically tracked to convert the distance measurements into a point cloud. A Conoprobe employs conoscopic holography, a distance measurement technique based on polarized light interference inside of a birefringent crystal [19], [20]. Since the 
Conoprobe captures one degree of freedom measurements along its laser axis, it is suitable for deployment through a laparoscopic port.

In this paper we apply this technology to guide a steerable active cannula robot using preoperative images. We describe the overall system and experiments designed to evaluate the accuracy with which the cannula tip can be positioned through a single insertion point to targets inside a soft tissue phantom model. This study lays the foundation for precise, steerable, image-guided access to locations within the human body for needle-based diagnoses and therapy delivery.

\section{A. System Concept}

Our Conoprobe-steerable cannula system consists of two primary sub-modules as shown in Fig. 1. Preoperative image data can be registered to the point cloud that the tracked Conoprobe collects, so that the positions of subsurface objects such as blood vessels, nerves, and tumors can be known in optical tracker space. Point cloud collection and registration is described in Section II. The second module is the steerable active cannula robot [10], [21], that controls the axial rotation and telescopic extension of a collection of precurved tubes (see Fig. 3). In Section III we give the inverse kinematics of the two-tube active cannula used in our experiments. The cannula actuation unit - which can be optically tracked or registered using the Conoprobe - deploys the cannula to a desired point within tissue, where needlebased therapy (e.g. injections, biopsies, thermal ablation, radioactive seed deployment, etc.) can be delivered.

\section{Surface Contour Sensing and Registration}

Surface contour sensing is accomplished using a tracked Conoprobe sensor as described in [18]. Our surface scans were acquired using the Conoprobe Mark 3 (Optimet, Inc.), with a $250 \mathrm{~mm}$ lens. While Optimet does not have published specifications for this particular lens, its performance is similar to that of the closest lens for which there are published specifications, a $200 \mathrm{~mm}$ lens, with which the device has a measurement precision of $<70 \mu \mathrm{m}$. The measurement range, which depends on focal length, was $155-337 \mathrm{~mm}$ for our study.

Optical tracking in our experiment was performed using a MicronTracker 2 H3-60 (Claron Technology, Inc). This is a passive optical tracking system that uses black and white

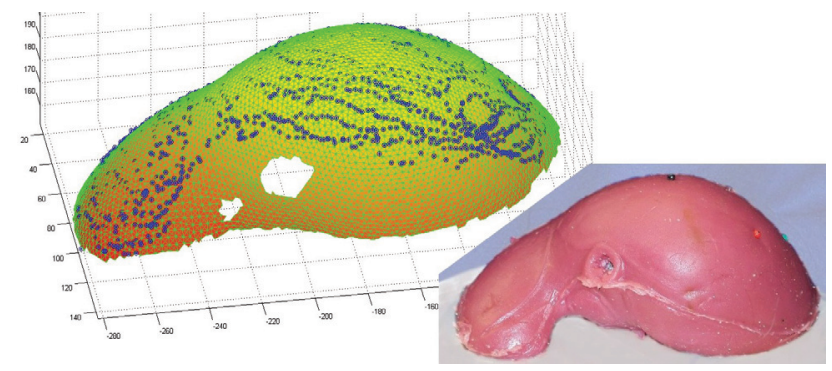

Fig. 2. Conoprobe-sensed point cloud fitted to the surface of the liver phantom, segmented from a CT scan. contrast markers, each sensed with an accuracy of $0.20 \mathrm{~mm}$, to create 6 DOF tracked markers.

The transformation between the Conoprobe's lens, the origin of the distance measurements, and its optical tracking marker frame is determined via calibration. The position of each sensed point on the surface can be expressed in the world (optical tracker) frame as,

$$
{ }^{\text {world }} p_{\text {sensed }}={ }^{\text {world }} T_{m}{ }^{m} T_{c}{ }^{c} p_{\text {sensed }},
$$

where $c$ denotes the Conoprobe lens frame and $m$ denotes the fiducial marker frame. The transformation ${ }^{\text {world }} T_{m}$ is given directly by the optical tracking system. We estimated ${ }^{m} T_{c}$ from several Conoprobe poses, with its laser aimed at a second fiducial marker which provided measurement of world $P_{n}$ by the optical tracking system. Repeating this for multiple Conoprobe poses, and parameterizing the rotation using $\mathrm{X}$ Y-Z Euler angles, we applied Matlab's fminsearch to minimize,

$$
\begin{aligned}
& e=\sum_{i} a_{i}^{\mathrm{T}} a_{i}, \text { where } \\
& \qquad a_{i}={ }^{\text {world }} p_{n}-{ }^{\text {world }} T_{m, i}{ }^{m} T_{c}{ }^{c} p_{\text {sensed }, i}
\end{aligned}
$$

over the Euler angles and translation that compose the unknown transformation. The sum over $i \in\{1 \ldots n\}$ accounts for each of the $n$ Conoprobe pose measurements made above.

With this system one can collect a cloud of points on the surface of the organ of interest. Given these and a segmented image, a number of strategies are possible to register the two, including both rigid and deformable techniques. We use Iterative Closest Point (ICP) [16] to perform a rigid registration between the surfaces. Figure 2 shows a sample segmented surface taken from a CT scan of a liver phantom after registration to a scanned point cloud of the same surface.

\section{Active Cannula Mechanics and Kinematics}

While active cannulas can be made from larger numbers of tubes, the protoype cannula we use in this study is composed of two concentric tubes. The outer tube is made of stainless steel and is straight. The inner tube is curved, and made from superelastic nitinol. The material and geometric properties of the two tubes used are summarized in Table I.

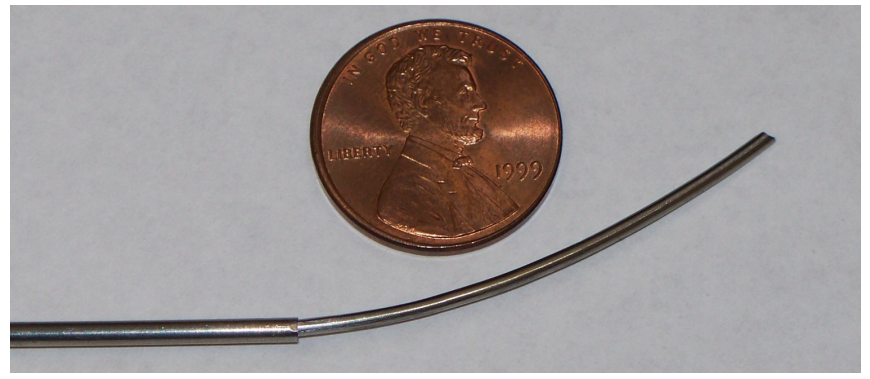

Fig. 3. Prototype active cannula consisting of a straight outer tube made of steel, and a precurved superlastic nitinol inner tube. 
TABLE I

Measured And Assumed Physical Quantities For EXPERIMENTAL CANNUla Tubes.

\begin{tabular}{|l|l|l|}
\hline & Inner Tube (1) & Outer Tube (2) \\
\hline Young's Modulus: $E(\mathrm{GPa})$ & 58 & 190 \\
\hline Inner Diameter: $r_{i}(\mathrm{~mm})$ & .96 & 1.52 \\
\hline Outer Diameter: $r_{o}(\mathrm{~mm})$ & 1.26 & 1.82 \\
\hline Straight Length: $L_{s}(\mathrm{~mm})$ & 255.7 & 171 \\
\hline Curved Length: $L_{c}(\mathrm{~mm})$ & 54 & 0 \\
\hline Total Length: $L(\mathrm{~mm})$ & 309.7 & 171 \\
\hline Curvature: $\kappa(1 / \mathrm{mm})$ & 0.0126 & 0 \\
\hline
\end{tabular}

\section{A. Cannula Mechanics}

We assume that the outer tube is initially straight, and that the transition point where the inner tube becomes curved lies between the constrained entry point and the end of the outer tube. Then, the prototype cannula, beyond the constrained entry point (see Fig 4), will consist of a straight section $\left(\ell_{1}\right)$ followed by a slightly curved section $\left(\ell_{2}\right.$ where the curved part of the inner tube bends the straight outer tube), followed by the curved portion of the inner tube alone after it exits the outer $\left(\ell_{3}\right)$. The plane in which the curved sections lie is controlled by the angular rotation of the tube bases, $\alpha$, and the length of each section is controlled by the translation of each tube's base. Our cannula, therefore, has 3 degrees of actuation freedom. Using the Bernoulli-Euler beam-mechanics model of [10], the curvature of the second section can be expressed as a "weighted average" curvature, weighted by the individual tube bending stiffnesses,

$$
\kappa_{e q}=\frac{\kappa_{1} E_{1} I_{1}+\kappa_{2} E_{2} I_{2}}{E_{1} I_{1}+E_{2} I_{2}} .
$$

where $E_{i}$ and $I_{i}$ are the elastic modulus and moment of inertia of tube $i$.

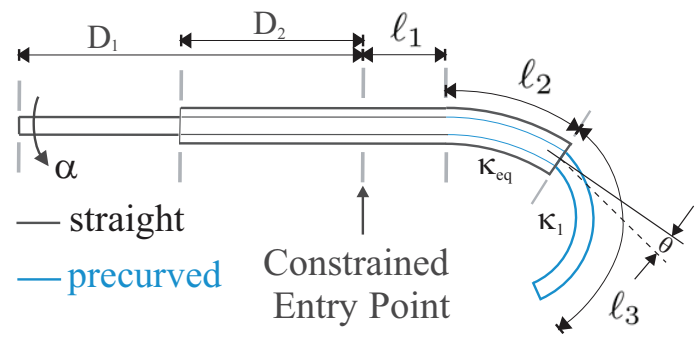

Fig. 4. Diagram of our two tube cannula showing kinematic variables.

\section{B. Cannula Kinematics}

Due to clearance between the tubes, the inner tube comes out of the outer tube at a slight angle to the tangent of the outer tube, which we denote $\theta$ (see Fig. 4). Prior models have not accounted for this non-tangency of arcs, but we include this effect in our kinematic model, experimentally measuring the angular offset $\theta$ to be $11^{\circ}$ using graph paper. The forward kinematic model then consists of a series of homogeneous transformations,

$$
T_{\text {tip }}=T_{\text {base }} T_{1} T_{2} T_{3} T_{4} T_{5}
$$

where the transformations $T_{i}$ are given by

$$
\begin{aligned}
& T_{1}=\left[\begin{array}{cccc}
\cos \alpha & -\sin \alpha & 0 & 0 \\
\sin \alpha & \cos \alpha & 0 & 0 \\
0 & 0 & 1 & 0 \\
0 & 0 & 0 & 1
\end{array}\right] \quad T_{2}=\left[\begin{array}{llll}
1 & 0 & 0 & 0 \\
0 & 1 & 0 & 0 \\
0 & 0 & 1 & \ell_{1} \\
0 & 0 & 0 & 1
\end{array}\right] \\
& T_{3}=\left[\begin{array}{cccc}
1 & 0 & 0 & 0 \\
0 & \cos \kappa_{e q} \ell_{2} & -\sin \kappa_{e q} \ell_{2} & \frac{1}{\kappa_{e q}}\left(1-\cos \kappa_{e q} \ell_{2}\right) \\
0 & \sin \kappa_{e q} \ell_{2} & \cos \kappa_{e q} \ell_{2} & \frac{1}{\kappa_{e q}} \sin \kappa_{e q} \ell_{2} \\
0 & 0 & 0 & 1
\end{array}\right] \\
& T_{4}=\left[\begin{array}{cccc}
1 & 0 & 0 & 0 \\
0 & \cos \theta & -\sin \theta & 0 \\
0 & \sin \theta & \cos \theta & 0 \\
0 & 0 & 0 & 1
\end{array}\right] \\
& T_{5}=\left[\begin{array}{cccc}
1 & 0 & 0 & 0 \\
0 & \cos \kappa_{1} \ell_{3} & -\sin \kappa_{1} \ell_{3} & \frac{1}{\kappa_{1}}\left(1-\cos \kappa_{1} \ell_{3}\right) \\
0 & \sin \kappa_{1} \ell_{3} & \cos \kappa_{1} \ell_{3} & \frac{1}{\kappa_{1}} \sin \kappa_{1} \ell_{3} \\
0 & 0 & 0 & 1
\end{array}\right]
\end{aligned}
$$

and the link lengths are given by,

$$
\begin{aligned}
& \ell_{1}=L_{s 1}-D_{1} \\
& \ell_{2}=L_{s 2}-D_{2}-\ell_{1} \\
& \ell_{3}=L_{c 1}-\ell_{2}
\end{aligned}
$$

where $D_{1}$ and $D_{2}$ are the translational kinematic inputs as shown in Fig. 4, and $L_{s i}$ and $L_{c i}$ are the straight and curved lengths of tube $i$.

\section{Cannula Inverse Kinematics}

We computed inverse kinematics numerically with Matlab's nonlinear root finding function fsolve, using the trust-region dogleg algorithm. The actuator values which result in a desired tip location and satisfy the physical constraints of the robot are the zeros of the following function,

$$
f\left(\alpha, D_{1}, D_{2}\right)=\left[\begin{array}{cl}
p_{\text {tip }}-p_{\text {desired }} & \\
D_{2}-D_{1} & \text { if } D_{1}<D_{2} \text {, else } 0 \\
D_{2} & \text { if } D_{2}<0, \text { else } 0
\end{array}\right]
$$

where $p_{t i p}$ is the position component of the final transformation $T_{t i p}$ from (4). The additional two components of (6) represent the physical constraints which ensure that the solver does not return a values of $D_{1}$ and $D_{2}$ which are impossible to achieve with the actuation unit.

\section{EXPERIMENTS}

A set of benchtop experiments was conducted to evaluate using Conoprobe surface scanning and preoperative image registration to guide a steerable active cannula to a desired target in an anthropomorphic human liver phantom made from cast silicone rubber. The experimental setup is illustrated in Figure 5, and consisted of the following components:

- Optimet Conoprobe. Tracked by the Micron Tracker, Conoprobe distance measurements were converted into a surface scan of the liver phantom. The power and frequency settings were $70 \%$ and $300 \mathrm{~Hz}$, respectively, as indicated by prior experiments [18].

- Micron Optical Tracker. Used to track the Conoprobe and an optical stylus. The optical tracker frame provides a common world coordinate frame in our system. 
- Aurora Magnetic Tracker. Is used to identify the location and orientation of the cannula tip via an embedded tracking coil. It is also used to sense the location of the target, a tracking coil embedded in the phantom. We note that this magnetic tracking system would not be present in an eventual clinical version of this system - here it simply provides an independent means of verifying accuracy.

- Needle with Embedded Magnetic Tracking Coil. This provided a target that could be inserted as desired into the phantom liver.

- Optical Stylus. An optically tracked pointer used to manually locate the cannula tip in free space during the registration of the cannula base frame to optical tracker. This would not be required in an eventual clinical system as the cannula actuation unit would either be directly optically tracked or located and registered via the Conoprobe system.

- Cannula Actuation Unit. A manually manipulated system that can accurately rotate and translate the cannula tubes. It consists of 2 rotary (Velmex small rotary table A5990T3) and 2 linear (Velmex model A25 series UniSlide linear slide) stages with resolutions of 0.1 degree and $0.01 \mathrm{~mm}$ respectively. A Matlab implementation of the cannula inverse kinematics model described in Section III yields the necessary rotational and linear positions of all four input degrees of freedom, given a desired tip position.

- Liver Phantom. A model of a human liver, cast in Smooth-On Ecoflex Supersoft 0010 silicone rubber. Four CT observable fiducials were attached to enable point-based registration to obtain an initialization frame for ICP registration.

- CT Scan and Segmentation Software. The liver phantom was CT scanned with a voxel size of $0.729 \times 0.729 \times$ $2 \mathrm{~mm}$. A level-set segmentation technique using Analyze 9.0 software (Mayo Clinic, Rochester, MN) and the marching cubes algorithm [22] contained within Kitware's Visualization Toolkit (VTK, Kitware Inc, www.vtk.org) yielded surface information. The surface was smoothed by fitting radial basis functions (RBF) using the FastRBF toolkit published by Farfield Technology (Christchurch, New Zealand) [23], [24]. Manual trimming and sampling of the RBF output yielded a point cloud representing only the upper surface of the phantom. The portions of the point cloud representing the bottom and internal portions of the phantom were removed since they would not be scanned by the Conoprobe. This subset point cloud, expressed in CT space, was used in all ICP registrations.

\section{A. Experimental Procedure}

The first step in the experiments was to register the coordinate systems to one another: Optical, CT, Magnetic, and Cannula base frame (see Fig. 7). The cannula base frame was registered to optical space via a point-based registration [25], [26]. The two point sets used for this each contained

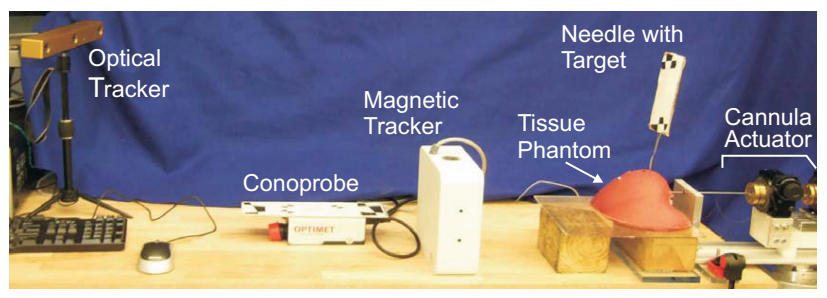

Fig. 5. Experimental setup.

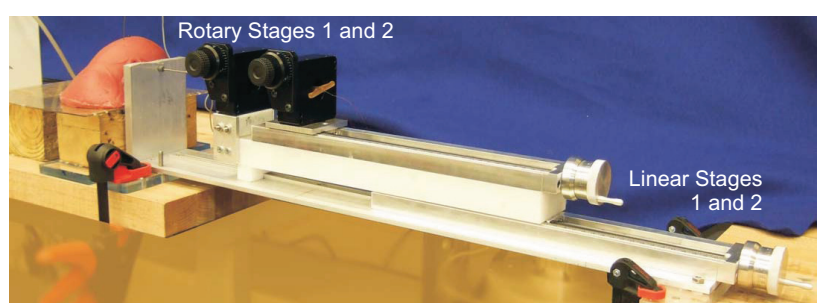

Fig. 6. Manual cannula actuation unit. The rotary stages independently rotate each tube axially. The primary linear stage, on the bottom, advances both tubes simultaneously. The secondary stage, on the top, advances only the inner tube and operates relative to the primary stage.

27 points. One set, expressed in the cannula base frame, was a set of 27 theoretical cannula tip positions, given by Equation 4, that spanned the cannula's workspace. These 27 positions consisted of all combinations of three values for the linear translations of the tubes, $\mathrm{D}_{1}$ and $\mathrm{D}_{2}\left(\mathrm{D}_{1}\right.$ : 166, 206, $246 \mathrm{~mm} ; \mathrm{D}_{2}:$ 79, 119, $159 \mathrm{~mm}$; see Fig 4), and three angular rotations spaced $120^{\circ}$ apart. The other point set was collected experimentally in the optical tracker frame, by using the optical stylus to record actual cannula tip positions at each of the 27 actuator positions used to generate the theoretical point set. The mean Euclidean error between these two point sets was $1.82 \mathrm{~mm}$. The end result of this calibration procedure is the frame transformation between the optical tracker frame and the cannula base frame.

After cannula base frame calibration, the cannula tubes were retracted and the phantom was positioned. CT space was then registered to the optical and magnetic coordinate frames via independent ICP fitting of a surface scan point cloud for each, in exactly the same manner as in [18]. The optical space cloud was collected using the Conoprobe. The magnetic space cloud was collected using a magnetically tracked touch probe that was lightly traced over the surface of the phantom. This method was adopted because it was more accurate than a fiducial based registration with the four fiducial markers implanted into the liver surface, due to segmentation error of the fiducials and the fact that the fiducials were spaced relatively close to each other. The fiducials simply provided the frame transformation from which to initialize ICP. To ensure that neither of these two independent ICP procedures converged to an incorrect local minimum (e.g. an "upside down" fit), a visual inspection of the final ICP fit (Fig. 2) was made to ensure correct alignment for every experiment in this paper. We note that in clinical practice, this visual inspection can be replaced or augmented by other techniques which provide a good initial guess from 


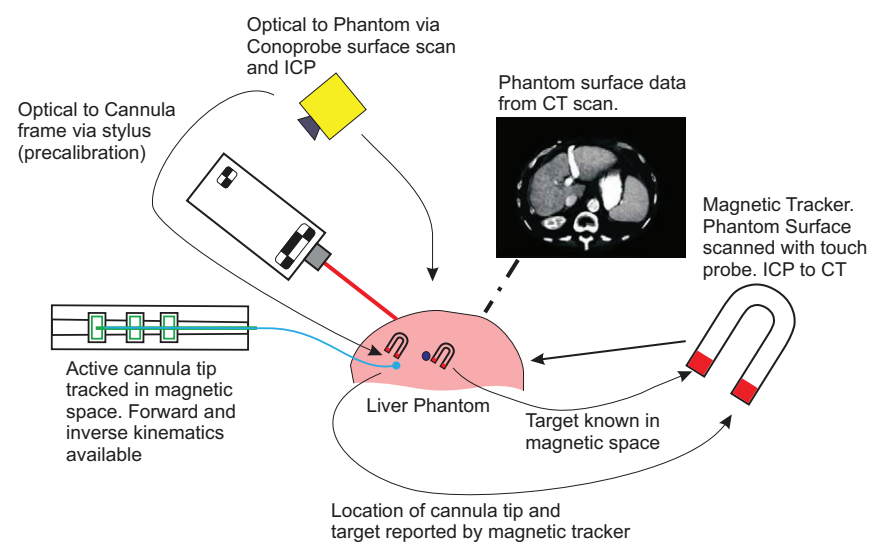

Fig. 7. Registrations in the system. The magnetic target is known in magnetic space. The cannula base frame is calibrated in optical space via a touch stylus, and the tip is tracked in magnetic space during insertion experiments and known in the cannula base frame via forward kinematics.

which to initialize the ICP registration [27].

After registration, the magnetic coil target (needle with embedded coil) was inserted into the phantom and its location was recorded in magnetic space. From magnetic space it was transformed into CT space, then to optical space, then to a location in the cannula base frame, and finally to a desired location in cannula actuator space using cannula inverse kinematics (Section III-C). The purpose of moving through the various frame transformations in this way was to simulate what we envision to be the eventual clinical workflow, wherein the target is specified by the physician in image space, and no magnetic tracker is used.

The cannula was then advanced into the liver phantom to the calculated actuator configuration. This was done by first adjusting rotary stages, and then deploying the translational degrees of freedom, beginning with the outer tube. After cannula insertion, the magnetic tracker was used to record tip position and final target position (note that the target generally shifted during insertion due to tissue deformation).

\section{B. Results}

The average difference between the cannula tip and target was $6.53 \mathrm{~mm}$ with a range of 5.42 to $8.87 \mathrm{~mm}$. The difference between the reported final location and targeted location averaged $8.32 \mathrm{~mm}$ with a range of 5.53 to $13.49 \mathrm{~mm}$. Experimental results for all 10 insertion experiments are reported in Table II.

\section{Discussion of Results}

Accuracies within 5-7 mm such as those we have obtained in these experiments are suitable for some medical procedures such as liver ablation. During ablation the treatment of large margins around a tumor is possible because of the organ's ability to regenerate. However, we believe that it will be possible to achieve significantly higher accuracies in the future. Below, we discuss various sources of error in our experiments and provide a perspective on which may be reduced, the accuracy improvement that can be expected, and how this might be accomplished in future work.
TABLE II

RESULTS OF 10 CANNULA PLACEMENT TRIALS

\begin{tabular}{c|c|c|c} 
Trial & $\begin{array}{c}\text { Cannula to } \\
\text { Target }(\mathbf{m m})\end{array}$ & $\begin{array}{c}\text { Cannula to } \\
\text { Original Target }(\mathrm{mm})\end{array}$ & $\begin{array}{c}\text { Target Shift } \\
\text { During Test }(\mathrm{mm})\end{array}$ \\
\hline $\mathbf{1}$ & $\mathbf{5 . 4 2}$ & 4.21 & 7.99 \\
$\mathbf{2}$ & $\mathbf{6 . 5 8}$ & 3.71 & 8.43 \\
$\mathbf{3}$ & $\mathbf{5 . 8 5}$ & 6.68 & 5.53 \\
$\mathbf{4}$ & $\mathbf{8 . 8 7}$ & 5.39 & 10.07 \\
$\mathbf{5}$ & $\mathbf{5 . 7 9}$ & 5.35 & 6.96 \\
$\mathbf{6}$ & $\mathbf{6 . 4 6}$ & 6.27 & 7.06 \\
$\mathbf{7}$ & $\mathbf{8 . 1 4}$ & 3.20 & 9.41 \\
$\mathbf{8}$ & $\mathbf{5 . 5 7}$ & 4.43 & 7.10 \\
$\mathbf{9}$ & $\mathbf{6 . 9 0}$ & 1.91 & 7.21 \\
$\mathbf{1 0}$ & $\mathbf{5 . 7 6}$ & 9.20 & 13.49 \\
\hline
\end{tabular}

1) Magnetic Tracking Error: We believe magnetic tracking error to be the largest source of error in our experiments, and there are a variety of ways it can introduce errors. First, the ICP surface registration and small deformations caused by use of the magnetic touch probe can introduce error into the magnetic to CT frame transformation. We believe these could be reduced by including a large number of widely spaced and easily segmented fiducials in a future phantom liver holder prior to $\mathrm{CT}$ scanning. These could enable a more accurate point-based rigid registration between CT and magnetic space. Second, there may also be small distortions in the magnetic field emitted by the tracker base station, which can be estimated using established methods [28]. We also note, that the magnetic tracking system used here was for verification of tip and target locations only, and would not be part of an eventual clinical system.

2) Optical Tracking Error: Uncertainty in optical tracking of the stylus and Conoprobe comes from the $0.20 \mathrm{~mm}$ RMS sensor noise in obtaining the position of each tracked marker. How this propagates to the tip of the pointer or measurement spot of the Conoprobe depends on how many markers are attached and the geometric relationship between the markers and the measurement point. To experimentally assess the amount of error these factors introduce in our system, we collected 200 measurements of the stylus and 200 measurements with the Conoprobe held stationary and aimed at a target $240 \mathrm{~mm}$ away (a typical scanning distance). The standard deviation of the stylus position error was $0.169 \mathrm{~mm}$, while the standard deviation of the Conoprobe was $0.159 \mathrm{~mm}$. Larger spacings and/or alternate arrangements of optical tracking markers can reduce these errors.

3) Optical ICP Error: ICP using Conoprobe measurements is intrinsic to our system, but collecting more points and/or more widely spaced points can improve accuracy. Toward understanding the quality of fit achieved in our experiments reported in this paper, we computed the mean residual error between the scanned points and each point's nearest neighbor in the segmented CT data. The Conoprobe surface alignment produced a mean residual error of $1.03 \mathrm{~mm}$ (the same calculation yields $3.03 \mathrm{~mm}$ for our magnetic surface registration). This can be compared to a mean residual error of $0.75 \mathrm{~mm}$ obtained with a commercial laser range scanner on a similar phantom [29]. 
4) Cannula Modeling Error: More advanced models of the cannula that do not assume circular curvature exist [21], and the latest models even account for external loading [30]. These may reduce registration error of the cannula base frame and lead to better predictions of cannula shape when interacting with tissue, potentially enhancing accuracy by several millimeters.

5) Tissue Deformation: Tissue deformation can be modeled (see e.g. [31]). Compensating for this would lead to further accuracy enhancements.

\section{CONCLusion}

In this paper, we have described a system that is able to acquire soft tissue surface data, register it to preoperative image data, and deliver the tip of a steerable cannula to physical locations that correspond to desired targets specified in preoperative image space. Combining the steerability of the active cannula with image guidance holds promise for enabling new and/or more accurate minimally invasive soft tissue procedures such as biopsy, tumor ablation, or other diagnostic or therapeutic agents and devices that can be delivered through a needle. Advantages of using preoperative images for guidance include the ability to perform image guided surgery without an intraoperative imaging, while making use of high quality preoperative images.

Furthermore, each of the technologies demonstrated in this experiment have potential in many other applications. Conoprobe enabled registration of the liver, kidney, or other organs could be used in conjunction with image guided laparoscopic surgeries. Active cannulas have been proposed for applications ranging from lung biopsies, brain surgery, and laparoscopic abdominal surgery under various forms of image guidance.

\section{REFERENCES}

[1] K. Cleary, A. Melzer, V. Watson, G. Kronreif, and D. Stoianovici, "Interventional robotic systems: applications and technology state-ofthe-art," Minimally Invasive Therapy \& Allied Technologies, vol. 15, no. 2, pp. 101-113, 2006.

[2] R. H. Taylor and D. Stoianovici, "Medical robotics in computerintegrated surgery," IEEE Trans. Robotics and Automation, vol. 19, no. 5, pp. 765-781, Oct. 2003.

[3] P. Potamianos, B. L. Davies, and R. D. Hibberd, "Intra-operative imaging guidance for keyhole surgery: methodology and calibration," First International Symposium on Medical Robotics and Computer Assisted Surgery, pp. 98-104, 1994.

[4] D. Stoianovici, R. J. Webster III, and L. Kavoussi, "Surgical robotic applications in minimally invasive uro-oncology surgery," in Minimally Invasive Uro-Oncologic Surgery, R. Moore and J. Bishoff, Eds. London and New York: Taylor and Francis, 2005, pp. 353-363, ISBN 1-84184-566-3.

[5] E. M. Boctor, M. A. Choti, E. Burdette, and R. J. Webster III, "3DUSguided robotic needle placement: An experimental evaluation," International Journal of Medical Robotics and Computer Aided Surgery, vol. 4, no. 2, pp. 180-191, 2008.

[6] S. P. DiMaio and S. E. Salcudean, "Needle steering and motion planning in soft tissues," IEEE Trans. Biomedical Engineering, vol. 52, no. 6, pp. 965-974, June 2005.

[7] D. Glozman and M. Shoham, "Image-guided robotic flexible needle steering," IEEE Trans. Robotics, vol. 23, no. 3, pp. 459-467, June 2007.

[8] S. Okazawa, R. Ebrahimi, J. Chuang, S. E. Salcudean, and R. Rohling, "Hand-held steerable needle device," IEEE/ASME Trans. on Mechatronics, vol. 10, no. 3, pp. 285 - 296, 2005.
[9] R. J. Webster III, J. S. Kim, N. J. Cowan, G. S. Chirikjian, and A. M. Okamura, "Nonholonomic modeling of needle steering," International Journal of Robotics Research, vol. 25, no. 5/6, pp. 509-526, May/June 2006.

[10] R. J. Webster III, J. M. Romano, and N. J. Cowan, "Mechanics of precurved-tube continuum robots," IEEE Trans. on Robotics, vol. 25, no. 1, pp. 67-78, 2009.

[11] P. Sears and P. E. Dupont, "A steerable needle technology using curved concentric tubes," IEEE/RSJ International Conference on Intelligent Robots and Systems, pp. 2850-2856, 2006.

[12] G. Fichtinger, T. L. DeWeese, A. Patriciu, A. Tanacs, D. Mazilu, J. H. Anderson, K. Masamune, R. H. Taylor, and D. Stoianovici, "System for robotically assisted prostate biopsy and therapy with intraoperative CT guidance," Academic Radiology, vol. 9, no. 1, pp. 60-74, 2002.

[13] J. Ding, D. Stoianovici, D. Petrisor, P. Mozer, R. Avila, L. Ibanez, W. Turner, D. Yankelvitz, E. Wilson, F. Banovac, and K. Cleary, "Medical needle steering for lung biopsy: Experimental results in tissue phantoms using a robotic needle driver," IEEE International Conference on BioInformatics and BioEngineering, 2008.

[14] N. V. Tsekos, A. Khanicheh, E. Christoforou, and C. Mavroidis, "Magnetic resonancecompatible robotic and mechatronics systems for image-guided interventions and rehabilitation: A review study," Annиal Review of Biomedical Engineering, vol. 9, pp. 351-387, 2007.

[15] M. I. Miga, T. K. Sinha, D. M. Cash, R. L. Galloway, and R. J. Weil, "Cortical surface registration for image-guided neurosurgery using laser-range scanning," IEEE Trans. on Medical Imaging, vol. 22, no. 8, pp. 973-985, 2003.

[16] P. J. Besl and N. D. McKay, "A method for registration of 3-D shapes," IEEE Trans. on Pattern Analysis and Machine Intelligence, vol. 14, pp. 239-256, 1992.

[17] W. R. Crum, T. Hartkens, and D. L. G. Hill, "Non-rigid image registration: theory and practice," The British Journal of Radiology, vol. 77, pp. s140-s153, 2004.

[18] R. A. Lathrop, D. Hackworth, and R. J. Webster III, "Minimally invasive holographic surface scanning for soft tissue image registration," IEEE Trans. on Biomedical Engineering, 2010, (In Press).

[19] G. Sirat and D. Psaltis, "Conoscopic holography," Optics Letters, vol. 10, no. 1, pp. 4-6, January 1985.

[20] G. Y. Sirat, F. Paz, G. Agronik, and K. Wilner, "Conoscopic systems and conoscopic holography," Optimet, Optimet White Paper.

[21] D. C. Rucker and R. J. Webster III, "Parsimonious evaluation of concentric-tube continuum robot equilibrium conformation," IEEE Trans. on Biomedical Engineering, vol. 56, pp. 2308-2311, 2009.

[22] W. E. Lorensen and H. E. Cline, "Marching cubes: A high resolution 3D surface construction algorithm," Computer Graphics, vol. 21, no. 4, pp. 163-169, 1987.

[23] J. C. Carr, W. R. Fright, and R. K. Beatson, "Surface interpolation with radial basis functions for medical imaging," IEEE Trans. On Medical Imaging, vol. 16, pp. 96-107, 1997.

[24] J. C. Carr, R. K. Beatson, J. B. Cherrie, T. J. Mitchell, W. R. Fright, B. C. McCallum, and T. R. Evans, "Reconstruction and representation of 3D objects with radial basis functions," in Proceedings of ACM SIGGRAPH, ser. Computer Graphics Proceedings, 2001, pp. 67-76.

[25] P. H. Schonemann, "A generalized solution of the orthogonal procrustes problem," Psychometrika, vol. 31, pp. 1-10, 1966.

[26] J. L. Farrell and J. C. Stuelpnagel, "Problem 65-1: A least squares estimate of satellite attitude," SIAM, vol. 8, pp. 384-386, 1966.

[27] L. W. Clements, D. M. Cash, W. C. Chapman, R. L. Galloway, and M. I. Miga, "Robust surface registration using salient anatomical features in image-guided liver surgery," Medical Physics, vol. 35, pp. $2528-40,2008$.

[28] C. Nafis, V. Jensen, L. Beauregard, and P. Anderson, "Method for estimating dynamic EM tracking accuracy of surgical navigation tools," Proceedings of the SPIE, vol. 6141, pp. 152-167, 2006.

[29] D. M. Cash, W. C. Chapman, H. Terawaki, B. M. Dawant, R. L. Galloway, and M. I. Miga, "Incorporation of a laser range scanner into image-guided liver surgery: Surface acquisition, registration, and tracking," Medical Physics, vol. 30, pp. 1671-1682, 2003.

[30] D. C. Rucker, B. A. Jones, and R. J. Webster III., "A geometrically exact model for externally loaded concentric tube continuum robots," IEEE Transactions on Robotics, (Accepted).

[31] R. Alterovitz, K. Y. Goldberg, J. Pouliot, and I.-C. Hsu, "Sensorless motion planning for medical needle insertion in deformable tissues," IEEE Trans. Information Technology in Biomedicine, vol. 13, no. 2, pp. 217-225, Mar. 2009. 